\title{
MEASUREMENTS AT LEP OF THE FORWARD-BACKWARD ASYMMETRIES OF QUARKS
}

Presented on behalf of the ALEPH, DELPHI, L3 and OPAL collaborations by:

Terry Wyatt,

Department of Physics, Schuster Laboratory,

The University, Manchester M13 9PL. U.K.

(Talk given at the International Conference on HEP, Dallas, Texas, USA. August 1992.)

\section{Abstract}

The measurements by the four LEP experiments of the forward-backward asymmetries of quarks are reviewed. In combining the measurements I have taken special care in the evaluation of common systematic errors that arise from our imprecise understanding of the production and decay of hadrons containing $\mathrm{b}$ and $\mathrm{c}$ quarks.

\section{INTRODUCTION}

The cross section for the production of quarkantiquark pairs in $\mathrm{e}^{+} \mathrm{e}^{-}$annihilation has the form:

$$
\frac{d \sigma}{d(\cos \theta)} \propto 1+\cos ^{2} \theta+b \cos \theta
$$

where $\theta$ is the angle between the incoming $\mathrm{e}^{-}$ and the outgoing quark. The number of 'forward' events $\left(N_{F}\right)$ is defined to be the number of events for which $\theta<\frac{\pi}{2}$. Similarly, $N_{B}$ is the number for which $\frac{\pi}{2}<\theta<\pi$. The term proportional to $\cos \theta$ in equation 1 leads to a 'forward-backward asymmetry' $\left(A_{\mathrm{FB}}\right)$ given by:

$$
A_{F B}=C\left(\frac{N_{F}-N_{B}}{N_{F}+N_{B}}\right)=\frac{3}{8} b
$$

If the angular range over which the asymmetry is measured is given by $|\cos \theta|<K$ then the correction factor $\mathrm{C}$ in equation 2 is given by:

$$
C=\frac{4 K}{3+K^{2}}
$$

In order to measure $A_{\mathrm{FB}}$ experimentally the jet originating from the quark must be distinguished from the jet originating from the antiquark. Ideally we should like to measure $A_{F B}$ for each flavour of quark individually. This requires the isolation of samples of multihadronic events that originate from the production of a single primary quark flavour and, in practice, this is possible only for $b$ and c quarks. The experimental techniques described in this report are: using the semileptonic decay $b \rightarrow 1$ to measure $A_{F B}^{b}$ (section 2) ; using the semileptonic decay $\mathrm{c} \rightarrow \mathrm{l}$ and tagged $\mathrm{D}^{* \pm}$ candidates to measure $\mathrm{A}_{\mathrm{FB}}^{\mathrm{c}}$ (section 3 ) ; using a momentum weighted jet charge to measure an average $A_{F B}$ without distinguishing between individual quark flavours (section 4).

$A_{F B}$ may be determined either by simply counting the number of forward and backward events or by fitting the observed angular distribution to the form given in equation 1 . A comparison of the results of the different methods provides a useful cross check of systematic biases. A fit to the angular distribution makes more efficient use of the available information, thus leading to a smaller statistical error. A maximum likelihood fit has the particular advantage that no knowledge of the experimental acceptance is required other than the fact that it is forward-backward charge symmetric. 
A chisquared fit, for which the data must be binned in $\cos \theta$ and corrected for any angular dependence of the experimental acceptance, allows a check to be made that the data are described by the angular distribution given in equation 1. For example figure 1 shows the results of a fit to angular distribution of $b$ quark events observed by the L3 experiment. (More details will be given in section 2).

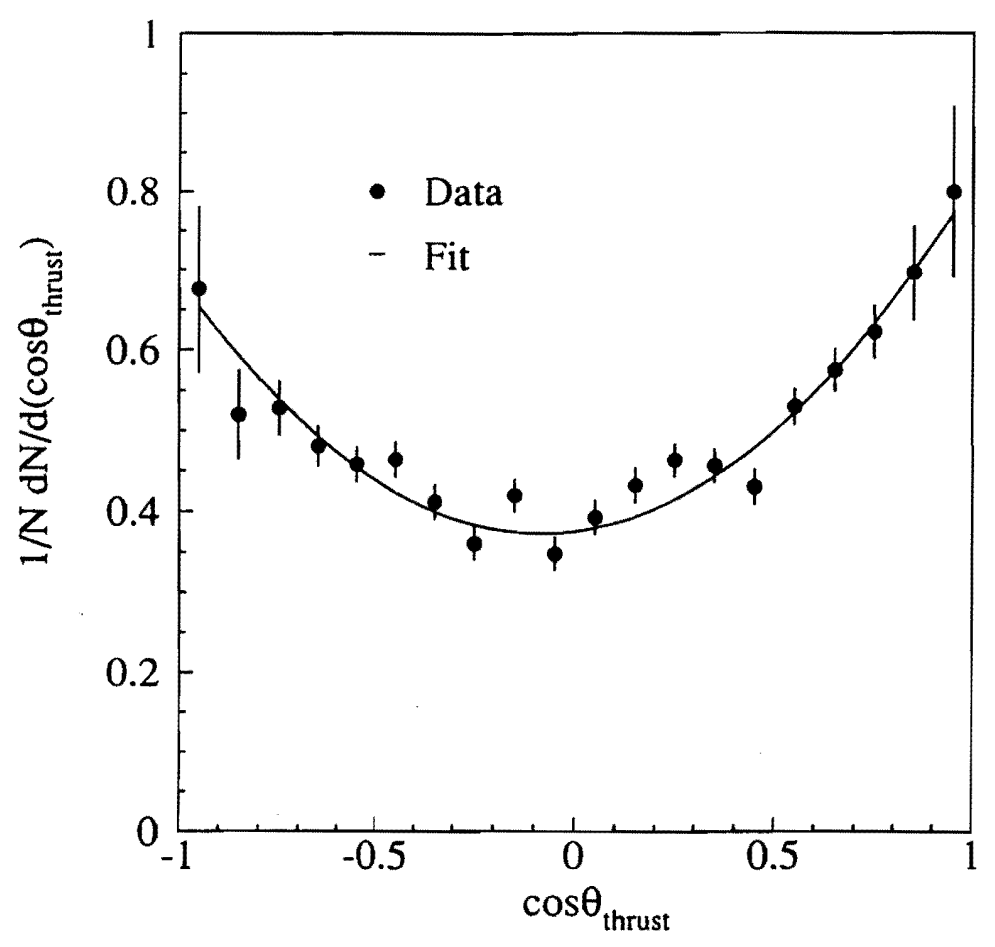

Figure 1: The angular distribution of $b$ quark events observed by the L3 experiment.

The $\mathrm{e}^{+} \mathrm{e}^{-}$collider LEP has so far been operated at centre of mass energies $\left(E_{\mathrm{cm}}\right)$ within $\pm 3 \mathrm{GeV}$ of the $\mathrm{Z}^{0}$ pole. The combined data sample collected by the four LEP experiments up to the end of 1991 , on which results are presented here, corresponds to about two million multihadronic $Z^{0}$ decays.

At $E_{c m}=M_{Z}$ the production of $q \bar{q}$ pairs is dominated by $\mathrm{Z}^{0}$ exchange and $\mathrm{A}_{\mathrm{FB}}$ is given in lowest order by:

$$
\mathrm{A}_{\mathrm{FB}}=3\left(\frac{a_{\mathrm{e}} v_{\mathrm{e}}}{a_{\mathrm{e}}^{2}+v_{\mathrm{e}}^{2}}\right)\left(\frac{a_{\mathrm{q}} v_{\mathrm{q}}}{a_{\mathrm{q}}^{2}+v_{\mathrm{q}}^{2}}\right)
$$

where $a_{\mathrm{e}}, v_{\mathrm{e}}$ are the axial and vector couplings of the electron and $a_{\mathbf{q}}, v_{\mathbf{q}}$ are the equivalent couplings for the quark flavour ' $q$ '. The main physics motivation for measuring $A_{F B}$ comes from the fact that it is sensitive to the effects of electroweak radiative corrections of the type shown, for example, in figure 2. The size
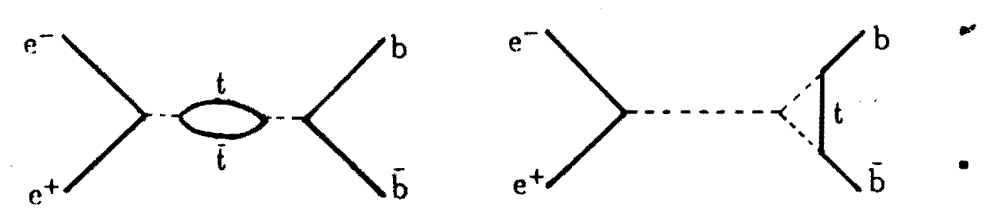

Figure 2: Examples of electroweak radiative corrections to $A_{F B}$.

of these corrections depends on the unknown mass of the top quark $\left(\mathrm{m}_{\text {top }}\right)$, as is shown for $\mathrm{A}_{\mathrm{FB}}^{\mathrm{b}}$ in figure 3 . It can be seen that in order

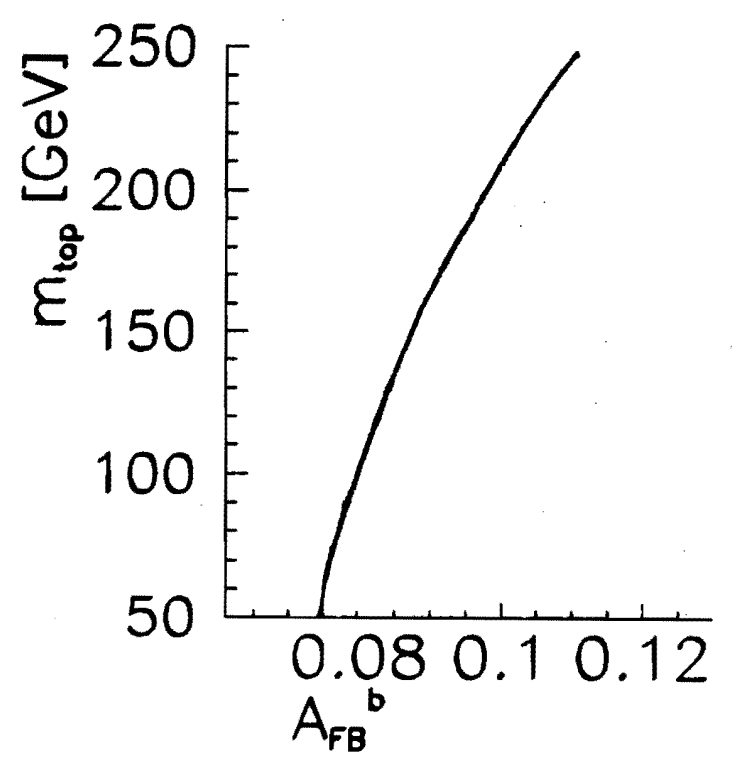

Figure 3: The dependence of $A_{F B}^{b}$ on $m_{\text {top. }}$

to achieve a sensitivity to $m_{\text {top }}$ at the level of $\sim 20 \mathrm{GeV}$ we need to measure $A_{\mathrm{FB}}^{\mathrm{b}}$ to an accuracy of \pm 0.005 or better.

\section{MEASUREMENT OF $\mathrm{A}_{\mathrm{FB}}^{\mathrm{b}}$}

All four experiments have used the semileptonic decay $b \rightarrow 1$ to measure $A_{F B}^{b}[1,2]$. Because of the hard fragmentation and large 


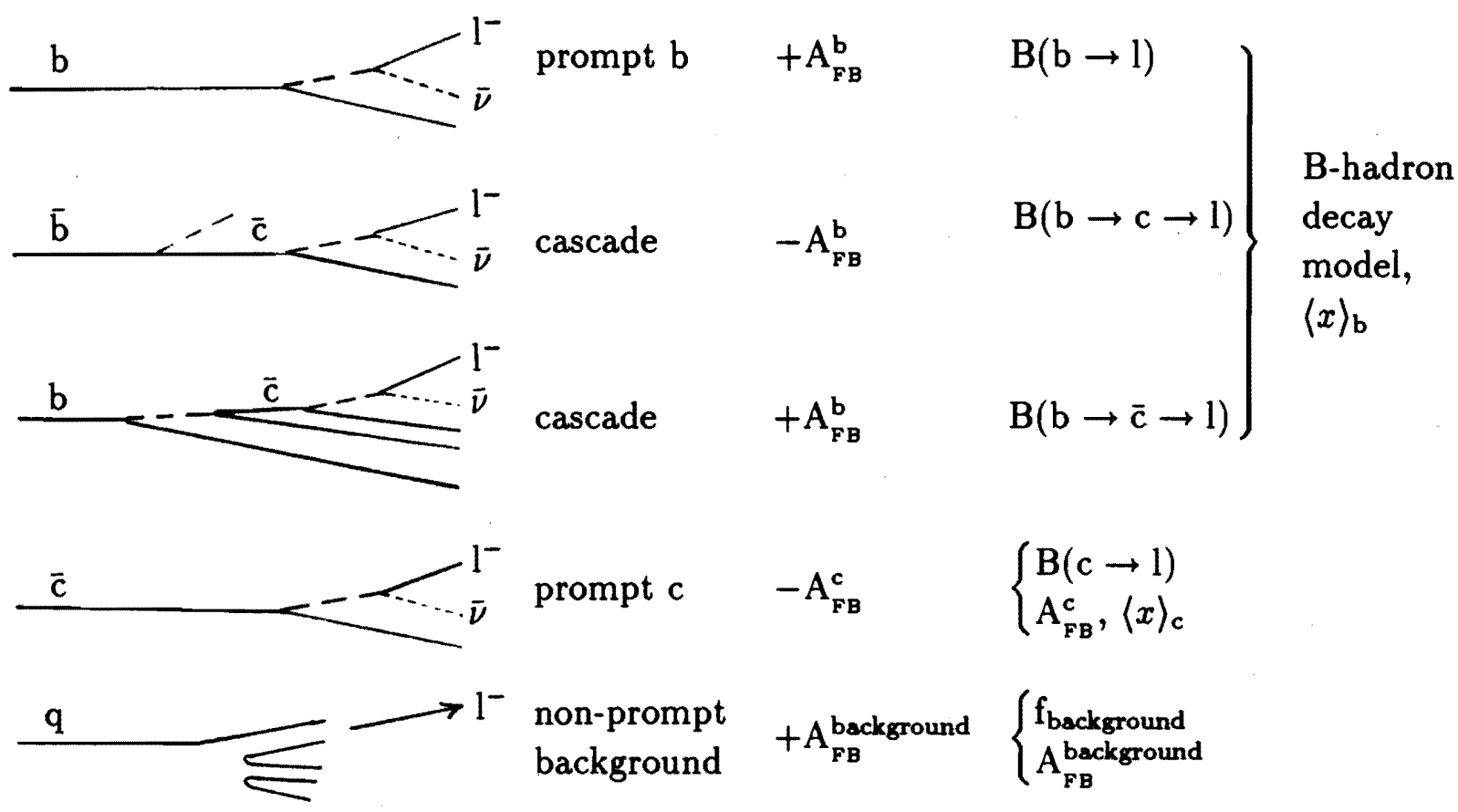

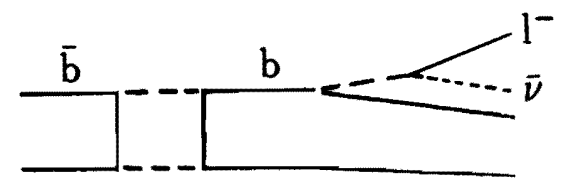

\section{$\mathrm{B}^{\circ} \overline{\mathrm{B}^{\circ}}$ mixing $-\mathrm{A}_{\mathrm{FB}}^{\mathrm{b}}$}

$\chi$

Figure 4: Contributions to the high $\mathrm{p}, \mathrm{p}_{\mathrm{T}}$ lepton sample.

mass of hadrons containing $b$ quarks, cuts in momentum ( $\mathrm{p} Z 3 \mathrm{GeV}$ ) and transverse momen-

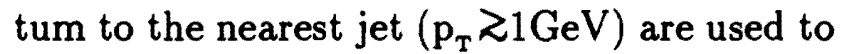
enhance the contribution from $b \rightarrow 1$. The sign of the lepton candidate enables $b$ and $\bar{b}$ jets to be distinguished. In order to estimate the production angle $\theta$ of equation 1 the thrust axis of the event is used; this is because compared to the direction of the jet containing the lepton the thrust axis is less sensitive to measurement errors and the effects of final state gluon bremsstrahlung.

In addition to the prompt semileptonic decay $b \rightarrow 1$, a number of other sources contribute to the high $\mathrm{p}, \mathrm{p}_{\mathrm{T}}$ lepton sample. These are shown schematically in figure 4 . The forward-backward asymmetry of the various contributions is shown in the third column of the figure. This leads to an experimentally observed asymmetry $\left(A_{F B}^{\text {raw }}\right)$ that is given by:

$$
\begin{aligned}
A_{F B}^{\text {raw }}= & \left(f_{b \rightarrow 1}-f_{b \rightarrow c \rightarrow 1}+f_{b \rightarrow \bar{c} \rightarrow 1}\right)(1-2 \chi) A_{F B}^{b} \\
& -f_{c \rightarrow 1} A_{F B}^{c}+f_{\text {background }} A_{F B}^{\text {background }}(5)
\end{aligned}
$$

where $f_{i}$ is the fraction of the high $p, p_{\mathrm{T}}$ lepton sample that originates from source ' $i$ ' and $\sum_{i} f_{i}=100 \%$. For the mixture of $B$ hadrons produced at LEP energies, $\chi$ is the average probability that a lepton from the direct $b \rightarrow 1$ decay of a $B$ hadron originated from a meson that had undergone $\mathrm{B}^{\circ} \overline{\mathrm{B}^{\circ}}$ mixing before decaying:

$$
\chi=\frac{\mathrm{B}\left(\mathrm{b} \rightarrow \mathrm{B}^{\circ} \rightarrow \overline{\mathrm{B}^{\circ}} \rightarrow 1\right)}{\mathrm{B}(\mathrm{b} \rightarrow 1)}
$$

It can be seen that uncertainties in the various terms in equation 5 will lead to systematic errors in the estimation of $A_{F B}^{b}$ from $A_{F B}^{\text {raw }}$. The relevant systematic uncertainties in $A_{F B}^{b}$ associated with each of the physical contributions 
to the high $p, p_{T}$ lepton sample are indicated in the fourth column of figure 4 .

I will first discuss the contribution of the non-prompt background. Highly redundant information for lepton identification is provided by the LEP experiments. For example, electrons may be identified using the specific energy loss $(d E / d x)$ in the tracking chambers, the transverse and longitudinal shape of the cluster of energy deposited in the calorimeters, and the matching in position and energy of the observed charged track and calorimeter cluster. The availability of several independent experimental signatures allows very detailed checks to be made of the relevant Monte Carlo simulations or, alternatively, allows the backgrounds and efficiencies to be determined using the data themselves. As an example we consider in figure 5 electron identification in the OPAL experiment. Figure 5 a) shows the $\mathrm{dE} / \mathrm{dx}$ distribution for tracks that pass all of the electron identification cuts other than the cut in $\mathrm{dE} / \mathrm{dx}$. The shaded region shows the fitted background contribution. The shape of the background was obtained from the data by selecting a sample of hadrons that had a transverse shower shape and deposited energy that was inconsistent with that expected for electrons. For comparison figure $5 \mathrm{~b}$ ) shows the deposited energy in the electromagnetic calorimeter divided by the measured momentum (E/p) for tracks that pass all of the electron identification cuts other than the cut in $\mathrm{E} / \mathrm{p}$. The shape of the background was obtained in this case by selecting a sample of hadrons that had $\mathrm{dE} / \mathrm{dx}$ inconsistent with that expected for electrons. The two independent estimates given by figure $5 \mathrm{a}$ ) and b) of the level of background remaining in the prompt electron signal are consistent. In a similar way the Monte Carlo predictions of the background in the prompt muon signal can be checked by using samples of pions from $\mathrm{K}^{0}$ decay in mul- tihadronic events and from 3-prong decays in tau pair events. Because of the wealth of available checks the experimental systematics are, in general, well under control and lead to errors that are small compared to the statistical errors. Because they depend on details of the individual experiments they are uncorrelated among the measurements from the four LEP collaborations.

In contrast to the above the remaining systematic uncertainties given in the fourth column of figure 4 reflect our imprecise understanding of the production and decay of hadrons containing $b$ and $c$ quarks. These uncertainties cause correlated systematic errors in the measurements from the four experiments, which must be taken into account when the results are combined.

The size of the correlated systematic errors will depend on the fractional composition $\mathrm{f}_{i}$ of the high $\mathrm{p}, \mathrm{p}_{\mathrm{T}}$ lepton samples. These are given for each of the four experiments in table 1 . It can be seen that the high $\mathrm{p}, \mathrm{p}_{\mathrm{T}}$ lepton

\begin{tabular}{|l|c|c|c|c|c|}
\hline & $\mathrm{l}$ & $\begin{array}{c}\mathrm{f}_{\mathrm{b} \rightarrow 1} \\
{[\%]}\end{array}$ & $\begin{array}{c}\mathrm{f}_{\mathrm{b} \rightarrow \mathrm{c}, \overline{\mathrm{c}} \rightarrow \mathbf{l}}[\%] \\
{\left[\begin{array}{c}\mathrm{f}_{\mathrm{c} \rightarrow 1} \\
{[\%]}\end{array}\right.}\end{array}$ & $\begin{array}{c}\mathrm{f}_{\text {background }} \\
{[\%]}\end{array}$ \\
\hline DELPHI & $\mathrm{e}$ & 53 & 12 & 11 & 24 \\
& $\mu$ & 63 & 11 & 9 & 17 \\
\hline L3 & $\mathrm{e}$ & 82 & 5 & 2 & 11 \\
& $\mu$ & 72 & 7 & 7 & 14 \\
\hline ALEPH & $\mathrm{e}$ & 85 & 7 & 4 & 4 \\
& $\mu$ & 79 & 7 & 4 & 10 \\
\hline OPAL & $\mathrm{e}$ & 78 & 10 & 6 & 6 \\
& $\mu$ & 77 & 7 & 4 & 12 \\
\hline \multicolumn{2}{|c|}{ 'typical' } & 80 & 7 & 4 & 9 \\
\hline
\end{tabular}

Table 1: Composition of the high $\mathbf{p}, \mathrm{p}_{\mathbf{T}}$ lepton samples.

samples of ALEPH, L3 and OPAL all have a similar composition. We can therefore expect that the correlated systematic errors on the extracted $A_{F B}^{b}$ values will be of a similar size for each of these samples. In order to estimate the size of these correlated errors I will 

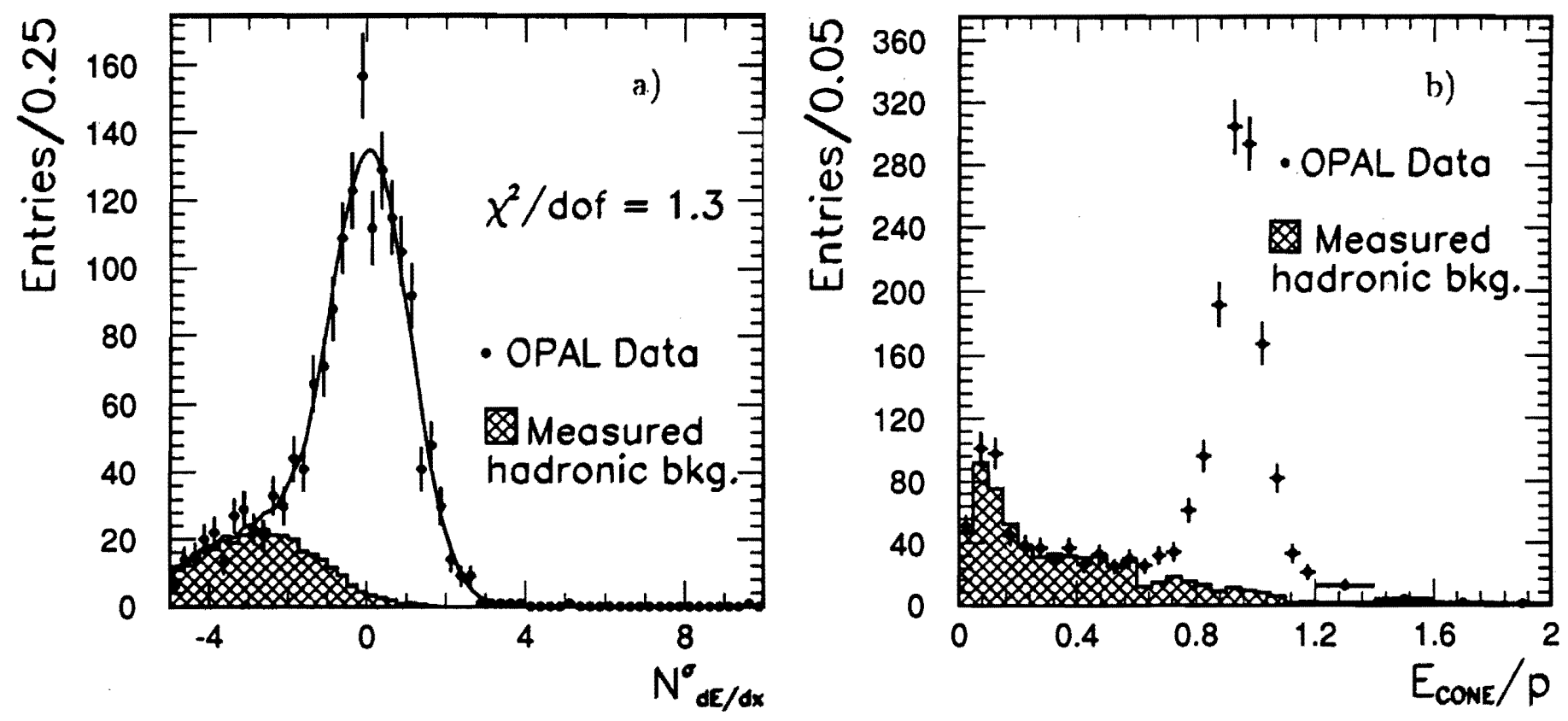

Figure 5: Background estimates in the inclusive electron analysis of OPAL: a) using dE/dx, b) using E/p.

consider a lepton sample corresponding to the 'typical' composition defined in the last row of table 1. This procedure obviously represents something of an approximation, but it is entrirely adequate given the current statisical precision of the measurements. The correlated systematic errors will be considered in two groups: those arising from our imprecise knowledge of the decay of $\mathrm{B}$ hadrons and those arising from the contribution of $\mathrm{Z}^{0} \rightarrow \mathrm{c} \overline{\mathrm{C}}$.

Our knowledge of B hadron semileptonic decay comes largely from measurements at the $\Upsilon_{48}$. For example, figure 6 shows the momentum spectrum of prompt leptons from $B$ meson decay measured by the CLEO experiment[3]. In order to extract the branching ratios $\mathrm{B}(\mathrm{b} \rightarrow \mathrm{l}), \mathrm{B}(\mathrm{b} \rightarrow \mathrm{c}, \overline{\mathrm{c}} \rightarrow \mathrm{l})$ from these data $\mathrm{a}$ prediction must be made for the momentum spectrum in the rest frame of the decaying $B$ hadron of leptons from these two processes. Table 2 shows the branching ratios extracted by CLEO[3], using the predictions of three different models of $B$ hadron decay. Note that the variations due to the models are large $(\sim 10 \%)$ and that the extracted values



Figure 6: The momentum spectrum of prompt leptons measured by CLEO.

of $\mathrm{B}(\mathrm{b} \rightarrow 1)$ and $\mathrm{B}(\mathrm{b} \rightarrow \mathrm{c}, \overline{\mathrm{c}} \rightarrow 1)$ are anticorrelated. Because the main cascade decay $b \rightarrow$ $c \rightarrow 1$ makes a contribution to the observed asymmetry that is opposite in sign to that of the primary decay $b \rightarrow 1$ (see equation 5 ) this effect amplifies the systematic error in the corrected value of $A_{F B}^{b}$. For $b \bar{b}$ events at LEP the shape of the lepton $\mathrm{p}_{\mathrm{T}}$ spectrum is determined largely by the momentum spectrum of leptons in the rest frame of the decaying $B$ 
hadron. The expected efficiency of a cut in $\mathrm{p}_{\mathrm{T}}$ will therefore also depend on which of the three $\mathrm{B}$ decay models is considered. Taking the correlated effects of the above uncertainties into account leads to a fractional error in the corrected asymmetry of: $\Delta \mathrm{A}_{\mathrm{FB}}^{\mathrm{b}} / \mathrm{A}_{\mathrm{FB}}^{\mathrm{b}} \approx 3 \%$.

\begin{tabular}{|c|c|c|c|}
\hline $\begin{array}{c}\text { branching ratio } \\
{[\%]}\end{array}$ & \multicolumn{3}{|c|}{ B decay model } \\
\cline { 2 - 4 } & ISGW & ACM & ISGW $^{* *}$ \\
\hline $\mathrm{B}(\mathrm{b} \rightarrow 1)$ & 9.9 & 10.5 & 11.2 \\
\hline $\mathrm{B}(\mathrm{b} \rightarrow \mathrm{c}, \overline{\mathrm{c}} \rightarrow \mathrm{l})$ & 11.3 & 9.7 & 9.0 \\
\hline
\end{tabular}

Table 2: B decay branching ratios measured by CLEO.

At LEP the cascade branching ratio $\mathrm{B}(\mathrm{b} \rightarrow \mathrm{c}, \overrightarrow{\mathrm{c}} \rightarrow 1)$ is expected to be lower than that measured by CLEO. This is because at the $\Upsilon_{4 s}$ only $B^{0}$ and $B^{ \pm}$mesons are produced, whereas at LEP we expect the production of also $B_{s}$ and $\Lambda_{b}$. These are expected to decay predominantly to $D_{s}$ and $\Lambda_{c}$, respectively, which have semileptonic branching ratios significantly smaller $(\sim 5 \%)$ than the average of $D^{0}$ and $D^{ \pm}(\sim 10 \%)$. If we assume:

$\mathrm{B}(\mathrm{b} \rightarrow \mathrm{c}, \overline{\mathrm{c}} \rightarrow 1)_{\mathrm{LEP}} \approx(.94 \pm .06) \mathrm{B}(\mathrm{b} \rightarrow \mathrm{c}, \overline{\mathrm{c}} \rightarrow 1)_{\Upsilon}$ and take into account the statistical and experimental systematic errors on the measurements of $\mathrm{B}(\mathrm{b} \rightarrow 1), \mathrm{B}(\mathrm{b} \rightarrow \mathrm{c}, \overline{\mathrm{c}} \rightarrow 1)$ at the $\Upsilon_{4 \mathrm{~s}}$ this leads to a fractional error in the corrected asymmetry of: $\Delta \mathrm{A}_{\mathrm{FB}}^{\mathrm{b}} / \mathrm{A}_{\mathrm{FB}}^{\mathrm{b}} \approx 2 \%$.

The contribution to $A_{\mathrm{FB}}^{\text {raw }}$ in equation 5 due to $Z^{0} \rightarrow c \bar{c}$ is $-f_{c \rightarrow 1} A_{F B}^{c}$. Assuming: the value $A_{F B}^{c}=0.072 \pm 0.027$ as calculated below in section 3 of this note; an uncertainty in the c quark fragmentation function corresponding to $0.54<\langle x\rangle_{c}<0.56$; and an uncertainty in the average semileptonic branching ratio $\Delta \mathrm{B}(\mathrm{c} \rightarrow \mathrm{l}) / \mathrm{B}(\mathrm{c} \rightarrow \mathrm{l}) \approx 15 \%$, leads to a fractional error in the corrected asymmetry of: $\Delta \mathrm{A}_{\mathrm{FB}}^{\mathrm{b}} / \mathrm{A}_{\mathrm{FB}}^{\mathrm{b}} \approx 3 \%$.

Within the minimal standard model the ratio $A_{F B}^{c} / A_{F B}^{b}$ is predicted to be almost inde- pendent of unknown parameters such as the masses of the top quark and Higgs particle. It is possible to use this relationship in order to reduce the systematic error on $A_{F B}^{b}$ coming from the prompt charm background. However, the resulting value of $A_{\mathrm{FB}}^{\mathrm{b}}$ can then be quoted only within the framework of the standard model. By using instead the experimentally measured value of $A_{F B}^{c}$, as was done above, the model dependence of the $A_{F B}^{b}$ result is minimized.

Combining the three errors given above gives a total correlated systematic error of $\Delta \mathrm{A}_{\mathrm{FB}}^{\mathrm{b}} / \mathrm{A}_{\mathrm{FB}}^{\mathrm{b}} \approx 5 \%$. The experimental measurements [1,2] of $A_{\mathrm{FB}}^{\mathrm{b}}$ are presented in the upper part of figure 7. The $A_{F B}^{b}$ values are not cor-

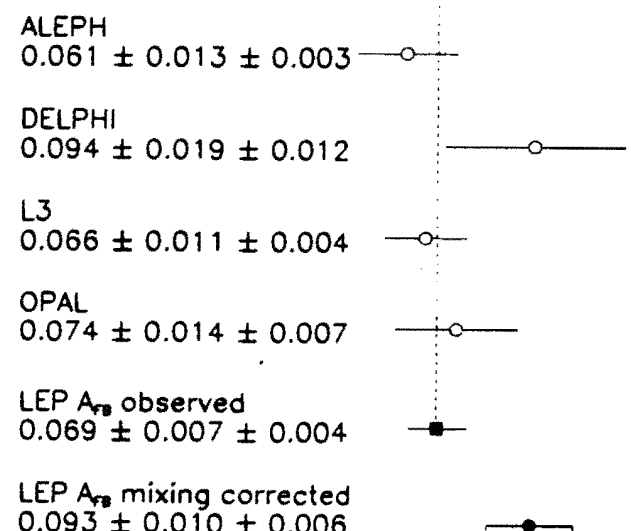
$0.093 \pm 0.010 \pm 0.006$

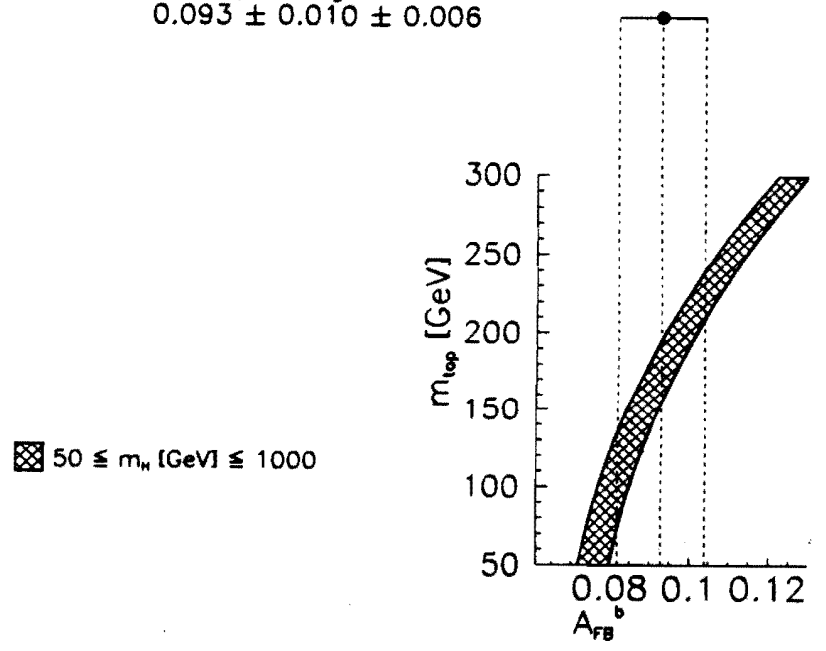

Figure 7: Summary of the results on $A_{F B}^{b}$.

rected for $\mathrm{B}^{\circ} \overline{\mathrm{B}^{\circ}}$ mixing. The first errors are statistical and the second are the systematic 
errors quoted by the experiments ${ }^{1}$. Removing from the systematic error of each experiment the contribution they assign to the sources considered above as correlated, gives the following uncorrelated systematic errors:

$\begin{array}{cccc}\text { ALEPH } & \text { DELPHI } & \text { L3 } & \text { OPAL } \\ \pm 0.002 & \pm 0.011 & \pm 0.004 & \pm 0.005\end{array}$

Combining the four measurements gives:

$$
\mathrm{A}_{\mathrm{FB}}^{\mathrm{b}}=0.069 \pm 0.007 \pm 0.004
$$

where the first error is the statistical and uncorrelated systematic and the second is the correlated systematic error corresponding to $\Delta \mathrm{A}_{\mathrm{FB}}^{\mathrm{b}} / \mathrm{A}_{\mathrm{FB}}^{\mathrm{b}} \approx 5 \%$ as calculated above.

Correcting for $\mathrm{B}^{\circ} \overline{\mathrm{B}^{\circ}}$ mixing using the combined LEP measurement $\chi=0.126 \pm 0.012$ [4] gives the final result:

$$
\mathrm{A}_{\mathrm{FB}}^{\mathrm{b}}=0.093 \pm 0.010 \pm 0.006
$$

where the uncertainty in the mixing correction has been added to the correlated error.

In the lower part of figure 7 the final result is compared to the prediction of the standard model as a function of $\mathrm{m}_{\text {top }}$. The experimental results are not corrected for electroweak and QCD radiative effects. These are, however, taken into account in the standard model prediction, which was obtained with the program ZFITTER [5]. A fit with this program yields the result: $m_{\text {top }}=176_{-68}^{+49} \mathrm{GeV}$, for $\mathrm{m}_{\text {higgs }}=300 \mathrm{GeV}, \alpha_{s}=0.12 . \quad$ Alternatively, the value of $\mathrm{A}_{\mathrm{FB}}^{\mathrm{b}}$ given above corresponds to: $\sin ^{2} \theta_{W}^{\text {eff }}=0.2321 \pm 0.0020$. The precision of this result is comparable to that obtained from the combined measurement of lepton pair forward-backward asymmetries at LEP,

${ }^{1}$ The $A_{F B}^{b}$ value from $L 3$ is obtained from a combined fit to $A_{F B}^{b}$ and $A_{F B}^{c}$. The error in $A_{F B}^{b}$ due to the uncertainty in $A_{F B}^{c}$ thus contributes to the statistical error of this fit. I have removed this contribution from the quoted L3 statistical error since it is considered above as a correlated systematic error. which gives: $\sin ^{2} \theta_{W}^{\text {eff }}=0.2320 \pm 0.0016$. However, whereas the latter measurement will be completely statistics limited for the forseeable future, we have seen above that systematic uncertainties in $A_{\mathrm{FB}}^{\mathrm{b}}$ are starting to become significant once the 1991 data of the four LEP experiments are combined. The 1992 running of LEP has more than doubled the available data samples. In order to make efficient use of these data, significant progress will have to be made in understanding some of the sources of systematic error discussed above. One way in which this may be achived is by the study of 'double tagged' samples, that is, events that contain two high $\mathrm{p}, \mathrm{p}_{\mathrm{T}}$ leptons or that contain a tagged $B$ decay vertex in addition to a high $\mathrm{p}, \mathrm{p}_{\mathrm{T}}$ lepton.

\section{MEASUREMENT OF $A_{\mathrm{FB}}^{\mathrm{c}}$}

Measurement of $A_{\mathrm{FB}}^{\mathrm{c}}$ using $\mathrm{c} \rightarrow 1$

Two experiments $[1,6]$ have measured $A_{\mathrm{FB}}^{c}$ using the semileptonic decay $c \rightarrow 1$. In general leptons from $c \rightarrow 1$ are produced at lower $p, p_{T}$ than those from $\mathrm{B}$ meson decay. The results:

$$
\begin{array}{lccc} 
& \mathrm{A}_{\mathrm{FB}}^{\mathrm{c}} & \text { (stat.) } & \text { (syst.) } \\
\text { ALEPH } & 0.064 & \pm 0.039 & \pm 0.030 \\
\text { L3 } & 0.083 & \pm 0.038 & \pm 0.027
\end{array}
$$

have been obtained from a combined fit to the $p, p_{T}$ spectrum that gives both $A_{F B}^{b}$ and $A_{F B}^{c}$. Because there are sizable backgrounds in the low $\mathrm{p}, \mathrm{p}_{\mathrm{T}}$ region both from non-prompt sources and from $B$ meson decay, the statistical and systematic errors are rather large. Of the

\begin{tabular}{|c|c|}
\hline correlated syst. errors & ALEPH \\
\hline 1$), \mathrm{B}(\mathrm{b} \rightarrow 1),\langle x\rangle_{\mathrm{c}},\langle x\rangle_{\mathrm{b}}$ & 0.017 \\
\hline$A_{F B}^{\text {background }}$ & 0.011 \\
\hline
\end{tabular}
sytematic errors quoted by the experiments I have considered the following two groups to be $100 \%$ correlated between the two measurements: 
This leaves uncorrelated systematic errors of: \pm 0.022 for ALEPH and \pm 0.012 for L3. The combined result is then:

$$
A_{\mathrm{FB}}^{c}=0.074 \pm 0.030 \pm 0.022
$$

where the first error is the statistical and uncorrelated systematic and the second is the correlated systematic error.

\section{Measurement of $A_{\mathrm{FB}}^{\mathrm{c}}$ using tagged $D^{* \pm}$}

Three experiments have used the low $Q$ value in the decay $D^{* \pm} \rightarrow D^{0} \pi^{+}$in order to tag $D^{* \pm}$ for measurents of $A_{F B}^{c}[2]$. The decay $\mathrm{D}^{0} \rightarrow \mathrm{K}^{-} \pi^{+}$leads to a narrow peak in the $\mathrm{K}^{-} \pi^{+}$mass spectrum at $\mathrm{m}_{\mathrm{D}^{0}}$. Figure 8 shows, for example, the $\mathrm{K}^{-} \pi^{+}$mass spectrum observed by the DELPHI experiment. In addition OPAL and DELPHI have used the decay $\mathrm{D}^{0} \rightarrow \mathrm{K}^{-} \pi^{+} \pi^{0}$, where the undetected $\pi^{0}$ leads to a broad peak in the $\mathrm{K}^{-} \pi^{+}$mass spectrum below $m_{D^{0}}$. This can also be seen in figure 8 .

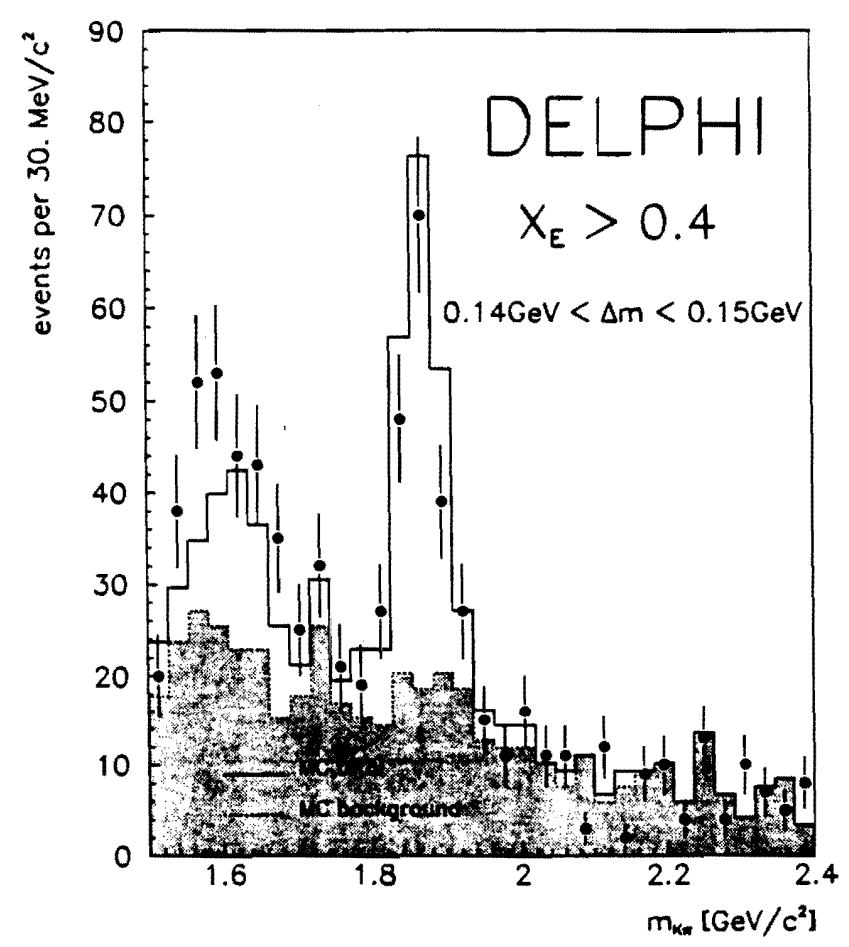

Figure 8: The $\mathrm{K}^{-} \pi^{+}$mass spectrum observed by the DELPHI experiment.

A cut in $x_{e}=2 E_{D} / E_{c m}$, where $E_{D}$ is the energy of the $D^{* \pm}$ candidates, is applied in or- der to distinguish between the relatively hard $\mathrm{D}^{* \pm}$ mesons that are produced by the fragmentation of primary $c$ quarks from the predominantly soft $\mathrm{D}^{* \pm}$ mesons that are produced from the decay of $B$ hadrons. The effectiveness of such a cut is illustrated by figure 9, which shows the $x_{e}$ distribution of $\mathrm{D}^{* \pm}$ candidates observed by the ALEPH experiment, compared with the distributions expected for the prompt $\mathrm{c}$ and $\mathrm{B}$ decay contributions.

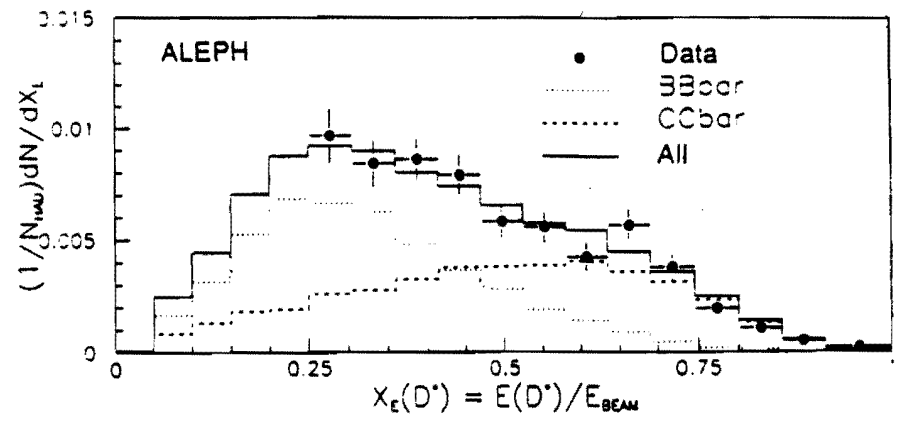

Figure 9: The $x_{\mathrm{e}}$ distribution of $\mathrm{D}^{* \pm}$ candidates observed by the ALEPH experiment, compared with the distributions expected for the prompt $c$ and $B$ decay contributions.

The available event samples are summarized in Table 3. The measured values of $A_{\mathrm{FB}}^{\mathrm{c}}$ are given in Table 4 . and presented as a function of $E_{c m}$ in figure 10. The statistical errors are much larger than the quoted systematic errors. In fact, the systematic errors are largely limited by statistics and will be substantially reduced once larger data samples are available. Combining the three measurements at the $\mathrm{Z}^{0}$ peak assuming no correlations yields the result:

$$
A_{\mathrm{FB}}^{c}=0.070 \pm 0.038
$$

In figure 11 the measurements of $A_{F B}^{c}$ from leptons and tagged $D^{* \pm} s$ are combined and the result:

$$
\mathrm{A}_{\mathrm{FB}}^{\mathrm{c}}=0.072 \pm 0.027
$$

is compared with the prediction of the standard model, which was obtained as a function of $m_{\text {top }}$ with the program ZFITTER [5]. It can 


\begin{tabular}{|r|c|c|c|c|c|c|}
\hline & $\mathrm{E}_{\mathrm{cm}}$ range & $\mathrm{D}^{0}$ mode & $x_{\mathrm{e}}$ range & $\begin{array}{c}\text { combinatorial } \\
\text { background } \\
\text { fraction [\%] }\end{array}$ & $\begin{array}{c}\text { background } \\
\text { subtracted } \\
\text { number } \mathrm{D}^{* \pm}\end{array}$ & $\begin{array}{c}\text { purity } \\
\mathrm{Z}^{0} \rightarrow \mathrm{c} \overline{\mathrm{c}} \\
{[\%]}\end{array}$ \\
\hline OPAL & all $\mathrm{E}_{\mathrm{cm}}$ & $\mathrm{K}^{-} \pi^{+}$ & $>0.5$ & 13 & 312 & 78 \\
& & $\mathrm{~K}^{-} \pi^{+} \pi^{0}$ & $>0.5$ & 47 & 385 & 78 \\
\hline ALEPH & peak $\pm 1 \mathrm{GeV}$ & $\mathrm{K}^{-} \pi^{+}$ & $>0.5$ & 5 & 296 & 78 \\
\hline DELPHI & peak only & $\mathrm{K}^{-} \pi^{+}$ & $>0.3$ & 30 & 188 & 54 \\
& & $\mathrm{~K}^{-} \pi^{+} \pi^{0}$ & $>0.4$ & 40 & 115 & 68 \\
\hline
\end{tabular}

Table 3: The tagged $\mathrm{D}^{* \pm}$ samples of ALEPH, DELPHI and OPAL.

\begin{tabular}{|l|c|c|c|c|}
\hline & $\mathrm{A}_{\mathrm{cB}}^{\mathrm{c}}$ & (stat.) & (syst.) & $\mathrm{E}_{\mathrm{cm}}$ range \\
\hline & 0.064 & \pm 0.049 & \pm 0.024 & peak only \\
OPAL & -0.085 & \pm 0.130 & \pm 0.032 & $\left\langle\mathrm{E}_{\mathrm{cm}}\right\rangle=89.7 \mathrm{GeV}$ \\
& 0.290 & \pm 0.100 & \pm 0.032 & $\left\langle\mathrm{E}_{\mathrm{cm}}\right\rangle=92.7 \mathrm{GeV}$ \\
\hline ALEPH & 0.045 & \pm 0.076 & \pm 0.004 & peak $\pm 1 \mathrm{GeV}$ \\
\hline DELPHI & 0.107 & \pm 0.075 & \pm 0.013 & peak only \\
\hline
\end{tabular}

Table 4: $A_{F B}^{c}$ from the tagged $D^{* \pm}$ 's of ALEPH, DELPHI and OPAL.



Figure 10: $A_{F B}^{c}$ from tagged $D^{* \pm}$ versus $E_{c m}$.

be seen that at the current level of precision the measurements of $A_{F B}^{c}$ do not provide very stringent tests of the standard model.

Currently, the two measurements of $A_{\mathrm{Fg}}^{c}$



Figure 11: Combined $\mathrm{l}^{ \pm}$and $\mathrm{D}^{* \pm}$ measurement of $A_{\mathrm{FB}}^{\mathrm{c}}$.

from leptons and tagged $D^{* \pm}$ 's contribute with roughly equal weight to the combined result. 
However, unless the systematic errors arising from the substantial model dependence of the $c \rightarrow 1$ measurement can be reduced it seems likely that the $D^{* \pm}$ measurement, which will be statistics limited for the forseeable future, will dominate the average once larger data samples become available.

\section{MEASUREMENT OF A $A_{\mathrm{FB}}$ USING JET CHARGES}

In quark fragmentation the leading hadrons carry information on the charge of the primary quark and tend to be produced with high momentum. Using this fact, three experiments have used a momentum weighted jet charge to distinguish, on a statistical basis, the jets originating from the positively and negatively charged quarks and thus measure $A_{\mathrm{FB}}[7]$. No attempt has been made to distinguish events originating from the different flavours of primary quarks.

Since the up-type and down-type quarks are expected to have an asymmetry of the same sign, but they have charges of opposite sign, there is a partial cancelation between the two types of quarks in the average charge asymmetry measured by such methods. Monte Carlo simulations are needed to evaluate the efficiency with which the correct charge assignment is made. The sensitivity of the calculated efficiency to details of the quark fragmentation model is the dominant source of systematic error in these analyses. This is estimated by comparing the predictions of the JETSET and HERWIG Monte Carlo programs and by varying the fragmentation parameters of the JETSET program within reasonable limits. The different measurements appear to be sensitive to different aspects of the fragmentation. For example, the largest single error in the ALEPH study comes from the diference between the JETSET and HERWIG predictions, whereas the OPAL result in almost completely insensitive to this difference. However, in combining the results I have chosen to treat the fragmentation errors quoted by the experiments as $100 \%$ correlated.

Unfortunately, not all the experiments quote a value of the average charge asymmetry corrected for the efficiency with which the correct charge assignment is made; this means that the only way the results can be directly combined is at the level of the extracted $\sin ^{2} \theta_{W}^{\text {eff }}$ values given below:

$\begin{array}{rccc} & \sin ^{2} \theta_{W}^{\text {eff }} & \text { (uncorrel.) } & \text { (correl.) } \\ \text { OPAL } & 0.2321 & \pm 0.0028 & \pm 0.0020 \\ \text { DELPHI } & 0.2345 & \pm 0.0030 & \pm 0.0027 \\ \text { ALEPH } & 0.2295 & \pm 0.0029 & \pm 0.0040 \\ \text { combined } & 0.2323 & \pm 0.0017 & \pm 0.0027\end{array}$

where the first error is the statistical and uncorrelated experimental systematic and the second is the correlated systematic error, which comes mainly from quark fragmentation, but also has a contribution from $\mathrm{B}^{\circ} \overline{\mathrm{B}^{\circ}}$ mixing. From the errors on the combined result given above, it is clear that unless significant progress can be made in controlling the fragmentation uncertainties such measurements have reached the limit of their accuracy and are no longer competitive with the direct measurement of $A_{\mathrm{FB}}^{\mathrm{b}}$ described in section 2 .

\section{SUMMARY}

Experimental measurements of the forwardbackward asymmetries of quarks made with the data collected at LEP up to the end of 1991 have been presented. The measurements of the four experiments have been combined taking into account correlated systematic errors. As a test of the standard model the most precise measurement, that of $A_{\mathrm{FB}}^{\mathrm{b}}$ using high $\mathrm{p}, \mathrm{p}_{\mathrm{T}}$ leptons:

$$
A_{F B}^{b}=0.093 \pm 0.010 \pm 0.006
$$


is currently of comparable precision to that obtained from the measurement of lepton pair forward-backward asymmetries. However, systematic uncertainties in $\mathrm{A}_{\mathrm{FB}}^{\mathrm{b}}$ are starting to become significant once the 1991 data of the four LEP experiments are combined.

At the current level of precision the measurements of $A_{\mathrm{FB}}^{\mathrm{c}}$ do not provide very stringent tests of the standard model. However, new measurements using tagged $\mathrm{D}^{* \pm}$ 's are largely statistics limited and look promising for the future. Measurements using a momentum weighted jet charge to measure an average $A_{F B}$ without distinguishing between individual quark flavours have now reached the limit of their accuracy unless significant progress can be made in controlling the uncertainties coming from fragmentation.

The 1992 running of LEP has more than doubled the available data samples for these analyses and results from these data are eagerly awaited.

Acknowledgements: I am very grateful to Bob Clare, Günter Quast, Dorothee Schaile, Vivek Sharma and Pippa Wells for their help during the preparation of this work.

\section{REFERENCES}

1. L3 Collaboration, CERN-PPE/92-121.

2. ALEPH, DELPHI, OPAL Collaborations, preliminary results submitted to the ICHEP conference, Dallas 1992.

3. CLEO Collaboration, Phys. Rev. D45 (1992) 2212.

4. To be published in the proceedings of the XII International Conference on Physics in Collision, Boulder, June 1992.

5. D. Yu. Bardin et al, Berlin-Zeuthen 89-08.

6. ALEPH Collaboration, Phys. Lett. B263 (1991) 325.
7. ALEPH Collaboration, Phys. Lett. B259 (1991) 377 , and preliminary results submitted to the ICHEP conference, Dallas 1992. DELPHI Collaboration Phys. Lett. B286 (1992) 201. OPAL Collaboration CERN-PPE/92-119. 